Article

\title{
Selenium-Nanoparticles-Loaded Chitosan/Chitooligosaccharide Microparticles and Their Antioxidant Potential: A Chemical and In Vivo Investigation
}

\author{
Kaikai Bai ${ }^{1,2, *,+}$, Bihong Hong ${ }^{1,2, \dagger}$, Wenwen Huang ${ }^{1,2}$ and Jianlin He $\mathrm{H}^{1,2}$ \\ 1 Third Institute of Oceanography, Ministry of Natural Resources, Xiamen 361005, China; \\ bhhong@tio.org.cn (B.H.); wwhuang@tio.org.cn (W.H.); hejianlin@tio.org.cn (J.H.) \\ 2 Technical Innovation Center for Utilization of Marine Biological Resources, Ministry of Natural Resources, \\ Xiamen 361005, China \\ * Correspondence: kkbai@tio.org.cn; Tel.: +86-592-2195309 \\ + The authors contributed equally to this work.
}

Received: 7 December 2019; Accepted: 31 December 2019; Published: 3 January 2020

\begin{abstract}
Selenium nanoparticles (SeNPs) have attracted attention due to their favorable properties, unique bioactivities, and potential for use in nutritional supplements and nanomedicine applications. However, the application of SeNPs in the clinic has been greatly hindered by their poor stability, and their potential to protect against alcohol-induced oxidative stress has not been fully investigated. Herein, SeNPs were synthesized in the presence of chitosan (CS) or chitooligosaccharide (COS), and a mixture of SeNPs, CS, and COS was spray-dried to prepare selenium-nanoparticles-loaded chitosan/chitooligosaccharide microparticles (SeNPs-CS/COS-Ms). Their physicochemical properties, including morphology, elemental state, size distribution, surface potential, and characteristic structure, were investigated. The release of SeNPs from the vehicle and the free radical scavenging ability of SeNPs-CS/COS-Ms were also studied. Furthermore, the safety of SeNPs-CS/COS-Ms and their antioxidant activity against alcohol were evaluated in mice. The results indicate that SeNPs-CS/COS-Ms, with a novel structure characterized by their smooth or wrinkled surface, hollow core, and COS body filled with SeNPs-CS nanobeads, were able to release SeNPs and scavenge DPPH and superoxide anion radicals. SeNPs-CS/COS-Ms were found to be much safer than selenite, and they might protect mice from ethanol-induced oxidative stress by reducing lipid and protein oxidation and by boosting glutathione peroxidase (GSH-Px), superoxide dismutase (SOD), and catalase (CAT). In conclusion, SeNPs-CS/COS-Ms offer a new way to develop stable SeNPs with higher efficacy and better biosafety, and the antioxidant potential of SeNPs-CS/COS-Ms against ethanol deserves further development.
\end{abstract}

Keywords: selenium; nano; chitosan; chitooligosaccharide; alcohol; antioxidant

\section{Introduction}

As a dietary nutrient, selenium (Se) is an indispensable trace element required for animals and humans, and plays an essential role in many aspects of health [1,2]. To meet the daily requirement of Se, Se supplementation is necessary, especially in those suffering from Se deficiency [1-3]. In recent years, selenium nanoparticles (SeNPs), a unique type of elemental selenium of nano-defined size, have attracted attention due to their favorable properties and excellent biological activities, such as scavenging free radicals $[4,5]$, antibacterial activity $[4,6]$, anti-tumor activity $[4,7]$, promoting animal growth $[4,8]$, boosting Se retention $[4,8,9]$, and enhancing oxidant status in vivo $[4,9-11]$. These 
nanoparticles seem to be more efficient than many Se compounds in boosting selenoenzymes, as they have lower acute toxicity as well as acceptable bioavailability [2,9-13]. SeNPs are regarded as a prospective Se supplement due to their potential for use in nutritional supplements, chemoprevention, chemical therapy against cancer, and other nanomedicine applications $[4,10,11]$.

The antioxidant activity of SeNPs plays an important part in the bioactivities of these nanoparticles, since Se is an integral part of the catalytic site of at least 25 human selenoproteins and enzymes and plays a role in protecting cells from oxidative injury [2,4]. Se deficiency might break the balance between oxidants and antioxidants in the body, which may increase oxidation-associated risks, especially when the body is challenged by oxidative stress [1,2,4,10-15]. However, animals and humans continue to suffer from various forms of oxidative damage induced by chemicals [16,17] or emotional stress [18,19]. Among the oxidation-associated risks, excessive consumption of alcohol can induce oxidative stress in the body [17,20], and Se deficiency can increase alcohol-induced oxidative stress in tissues $[10,11,17,21]$. However, the protective potential of SeNPs against alcohol-induced oxidative challenge has yet to be reported. In addition, the commercial application of SeNPs in oral administration systems has been hindered by their poor stability [10,11]. A feasible formulation of SeNPs needs to be developed.

Chitosan (CS) is the only positively charged natural polysaccharide and has excellent biodegradability and biocompatibility [22]. The polysaccharide and its derivatives have been extensively examined in the pharmaceutical industry due to their potential in the development of medicine delivery systems [22]. In this study, SeNPs were quickly synthesized in CS, and a mixture of SeNPs, CS, and chitooligosaccharide (COS) was spray-dried to produce selenium-nanoparticles-loaded chitosan/chitooligosaccharide microparticles (SeNPs-CS/COS-Ms). The structure, SeNP release performance, free radical scavenging ability, and biosafety of the SeNPs-CS/COS-Ms were investigated, and the antioxidant potential of this new Se formulation was also studied in mice given alcohol. The authors hope that the results of this study will assist with the development of a SeNP-based Se supplement.

\section{Materials and Methods}

\subsection{Materials and Animals}

\subsubsection{Materials}

Food-grade CS (90.32\% deacetylated, an average molecular weight of $37 \mathrm{kDa}$ ) and COS (an average molecular weight of $2.5 \mathrm{kDa}$ ) were purchased from Aoxin Pharmaceutical Co. Ltd. (Taizhou, China). DPPH (1,1-diphenyl-2-picrylhydrazyl), pepsin from porcine gastric mucosa ( $\geq 250 \mathrm{units} / \mathrm{mg}$ solid), pancreatin from porcine pancreas $(8 \times$ United States Pharmacopoeia (USP) specifications), and pyrogallic acid of high purity were obtained from Sigma-Aldrich (St.Lousi, MO, USA). Reagents of food grade, including acetic acid, sodium selenite, and ascorbic acid ( $\mathrm{Vc}_{\mathrm{c}}$ ), and other reagents of analytical grade, were purchased from commercial suppliers (Sinopharm Chemical Reagent Co., Ltd., Shanghai, China). The assay kits for measuring thiobarbituric-acid-reactive substances (TBARS), glutathione (GSH), glutathione peroxidase (GSH-Px), superoxide dismutase (SOD), catalase (CAT), total carbonyl compounds (TCCs), and protein content were provided by Jiancheng Bio-engineering Institute (Nanjing, China).

\subsubsection{Animals}

Kunming (KM) mice of specific-pathogen-free (SPF) grade, half male and half female, 8-10 weeks old, and weighing 18-22 g, were supplied and housed by the Laboratory Animal Center, Shenyang Pharmaceutical University (Shenyang, China) with the license No. SCXK (Liaoning) 2015-0001. In addition, male KM mice of SPF grade, 8-10 weeks old, and 20-25 g in body weight (bw) were purchased from Beijing HFK Bioscience Co., Ltd. with the license No. SCXK (Beijing) 2014-0004, and were housed in a standardized sterile animal room located at the Fujian Health College (Fuzhou, 
China) with a controlled temperature $\left(25 \pm 2{ }^{\circ} \mathrm{C}\right)$ and humidity $(50 \pm 10 \%)$ and a 12-h light/dark cycle. The procedures that were utilized in the animal experiments were approved by the Animal Ethics Committees at Shenyang Pharmaceutical University (ethical committee approval number: SYPU-IACUC-C2017-4-21-208, Date (21/4/2017)) and Fujian Health College (ethical committee approval number: DW2018112501, Date (25/11/2018)). They were also compliant with the provisions and general recommendations of the Chinese Experimental Animals Administration Legislation.

\subsection{Chemicals and Characterization}

\subsubsection{Synthesis and Characterization of SeNPs}

SeNPs were synthesized in the presence of CS or COS as described in previous studies $[10,11]$ with little modification. Chitosan-decorated selenium nanoparticles (CS-SeNPs) were dialyzed against $1 \%(w / w)$ acetic acid to remove the excess Vc and other by-products. The purification of CS-SeNPs was conducted by means of ultrafiltration (UF) in an acetic acid solution, unless the permeate did not fade in $1 \mu \mathrm{mol} / \mathrm{L} \mathrm{KMnO}_{4}$ solution within $20 \mathrm{~min}$. The morphological characteristics of SeNPs were observed by using a TEM (JEM-2100; JEOL, Tokyo, Japan) device equipped with an energy-dispersive X-ray spectroscopy (EDS) machine as previously stated $[10,11]$. The size distribution and the zeta-potential of SeNPs were measured by using a Zetasizer Nano ZS particle analyzer (VEM3600; Malvern Instruments, Malvern, UK) with a $173^{\circ}$ scattering angle as previously described $[10,11]$.

\subsubsection{Preparation and Characterization of SeNPs-CS/COS-Ms}

The purified CS-SeNPs were mixed with COS or additional CS, and the mixture was spray-dried using a laboratory spray dryer (SY-6000; Shiyuan Biological Equipment Engineering Co. Ltd., Shanghai, China) with a standard $0.7 \mathrm{~mm}$ nozzle to generate SeNPs-CS/COS-Ms. The proportions of Se, CS, and COS could be adjusted by modifying the material ratio, which also allowed for the preparation of $\mathrm{CS} / \mathrm{COS}$ microparticles (namely CS/COS-Ms) without SeNPs. The Se content of SeNPs-CS/COS-Ms was determined by utilizing inductively coupled plasma mass spectrometry (ICP-MS) [23].

The morphology of SeNPs-CS/COS-Ms was observed by an SEM (S-4800; Hitachi, Tokyo, Japan) device. The size distribution of these microspheres was measured by using a particulate size analyzer (LS-POP(6); Zhuhai OMIC Instruments Co. Ltd., Zhuhai, China) $[10,11]$. In order to explore structural details, the ultrasonic disruption of SeNPs-CS/COS-Ms was performed in absolute ethanol or $50 \%(v / v)$ ethanol before SEM observations were made. Moreover, a Nicolet Nexus 470 spectrometer (Thermo Fisher Scientific, Waltham, MA, USA) was used to determine the FTIR spectra, which were acquired at $400-4000 \mathrm{~cm}^{-1}$ with a $4 \mathrm{~cm}^{-1}$ resolution $[10,11]$. In addition, an XPS measurement was performed using a high-resolution photoelectron spectrograph (Escalab 250Xi; Thermo Fisher Scientific, Waltham, MA, USA) equipped with a monochromatic $\mathrm{Al} K_{\alpha} \mathrm{X}$-ray source and a dual-beam charge neutralization system composed of a low-energy electron flood gun $(\sim 1 \mathrm{eV})$ and an argon ion gun $(\leq 10 \mathrm{eV})[10,11]$.

\subsection{Release Experiment}

The release of SeNPs from SeNPs-CS/COS-M-C was evaluated in simulated gastric fluid (SGF) [24] composed of $\mathrm{HCl}$ solution ( $\mathrm{pH} 1.5)$ with $0.1 \%(w / v)$ pepsin and simulated intestinal fluid (SIF) [25] composed of phosphate-buffered saline (PBS) ( $\mathrm{pH} 7.2)$ with $1.0 \%(w / v)$ pancreatin. Briefly, $0.1 \mathrm{~g}$ of SeNPs-CS/COS-Ms was placed in $100 \mathrm{~mL}$ of SGF or SIF, and the mixture was stirred at $100 \mathrm{rpm}$ and incubated at $37^{\circ} \mathrm{C}$. At certain time intervals, $1 \mathrm{~mL}$ of release medium was collected and $1 \mathrm{~mL}$ fresh SGF or SIF was placed in the release system. The medium was centrifuged $(1000 \times g, 1 \mathrm{~min})$, and then the supernatant was filtrated by using a PES filter membrane (with a pore size of $0.22 \mu \mathrm{m}$ ) to obtain the filtrate containing released SeNPs. The Se content of the filtrate was measured by an ICP-MS assay according to the method of Dufailly [23]. The Se contents were plotted as a function of time. All experiments were performed in triplicate. 


\subsection{Free Radical Scavenging Tests}

\subsubsection{DPPH Radical (•DPPH) Scavenging Assay}

The •DPPH scavenging assay was performed according to the method of Zhai [26] and Xu [27], with a slight modification. In brief, a sample was mixed with $1 \mathrm{~mL}$ of DPPH ethanol solution $(1 \mathrm{mM})$, and the mixture was adjusted to $10 \mathrm{~mL}$ using ethanol. After incubation for $30 \mathrm{~min}$ at $25^{\circ} \mathrm{C}$, the absorbance of the mixture was measured at $517 \mathrm{~nm}$. The DPPH radical scavenging ability was calculated as follows:

$$
\text { Scavenging ability }(\bullet \mathrm{DPPH})=\left(\mathrm{A}_{\mathrm{c}}-\mathrm{A}_{\mathrm{a}}+\mathrm{A}_{\mathrm{b}}\right) / \mathrm{A}_{\mathrm{c}} \times 100 \%
$$

where $A_{c}$ is the absorbance of DPPH without a sample; $A_{a}$ is the absorbance of the sample mixed with DPPH solution; and $A_{b}$ is the absorbance of the sample without DPPH solution.

\subsubsection{Superoxide Anion Radical $\left(\bullet \mathrm{O}_{2}^{-}\right)$Scavenging Assay}

The influence of a sample on the generation of $\bullet \mathrm{O}_{2}{ }^{-}$by pyrogallic acid [28] was determined by using a spectrophotometric method with some modifications. Tris- $\mathrm{HCl}$ buffer $(50 \mathrm{mM}, \mathrm{pH} 8.2)$ containing certain concentrations of the tested sample was incubated at $25^{\circ} \mathrm{C}$ for $30 \mathrm{~min}$. Subsequently, it was mixed with pyrogallic acid $(0.75 \mathrm{mM})$ and rapidly shaken. The mixture was quickly adjusted to the same volume, and its absorbance was recorded in succession using a spectrophotometer (UV-1780, Shimadzu, Kyoto, Japan) at $320 \mathrm{~nm}$. The slope value of each absorbance curve was measured through linear regression. The ability to scavenge $\bullet \mathrm{O}_{2}{ }^{-}$was calculated as follows:

$$
\text { Scavenging ability }\left(\bullet \mathrm{O}_{2}^{-}\right)=\left(\mathrm{S}_{\mathrm{a}}-\mathrm{S}_{\mathrm{b}}\right) / \mathrm{S}_{\mathrm{a}} \times 100 \%
$$

where $S_{a}$ was the slope rate when the sample was mixed with pyrogallic acid; and $S_{b}$ was the slope rate when only pyrogallic acid was used.

\subsection{Animal Experiment}

\subsubsection{Acute Lethal Test In Vivo}

The acute lethal property of SeNPs-CS/COS-Ms (CS:COS = 10:90 w/w, containing $15.1 \mathrm{mg}$ Se/kg) was determined as previously described [10,11] with little modification. Briefly, 50 KM mice were randomly divided into five groups with 10 mice per group after adaptation for 3 days. Each group was given SeNPs-CS/COS-Ms (10.00, 7.50, 5.62, 4.20, and $3.16 \mathrm{~g} / \mathrm{kg}$ bw, respectively) by a single intragastric administration, and cumulative mortality within 14 days was recorded to calculate the median lethal dose (or $\mathrm{LD}_{50}$ ) by the Bliss method [29]. Another group of $40 \mathrm{KM}$ mice was given CS/COS-M (CS:COS = 10:90 w/w) at a cumulative dose of $20 \mathrm{~g} / \mathrm{kg}$ bw within $12 \mathrm{~h}$ to evaluate the acute toxicity of this vehicle for SeNPs.

\subsubsection{Ethanol Challenge Test}

An alcohol challenge test was performed as described by Han [30] and Zeng [31] with some modifications. In brief, 80 male KM mice were randomly divided into eight groups (10 mice per group) (Control, Model, Vc, Selenite, CS/COS-M (CS:COS = 10:90), and three SeNPs-CS/COS-Ms (CS:COS = 10:90, containing $15.1 \mathrm{mg} \mathrm{Se} / \mathrm{kg}$ ) groups (L-Se, M-Se, and H-Se)). Each group was administered a dose daily by a gavage for five consecutive weeks, as shown in Table 1, and body weight was recorded throughout the whole experiment. Except for the normal control, mice were deprived of food after the final pretreatment, but were allowed free access to water. Sixteen hours later, normal control mice were given an equal volume of water and the fasting mice were intragastrically administered a single dose of $50 \%$ ethanol ( $4.8 \mathrm{~g} / \mathrm{kg}$ bw, namely $12 \mathrm{~mL} / \mathrm{kg}$ bw). Six hours later, the mice were sacrificed, the blood was collected into heparin-free tubes to obtain serum, and the liver was also 
obtained. The Se content in serum was determined by an ICP-MS assay [23]. Then, the GSH-Px, SOD, CAT, TBARS, GSH, and TCC levels in serum or in liver were measured, following the instructions in the commercial kits.

Table 1. Intragastric administration during the ethanol challenge experiment (Kunming (KM) mice, $n=10)$.

\begin{tabular}{ccc}
\hline Group & $\begin{array}{c}\text { Pre-Treatment (Once Daily, Day } \\
\text { 1-35) }\end{array}$ & Treatment (Once, Day 36) \\
\hline Control & Normal saline & Normal saline \\
Model & Normal saline & $50 \%$ Ethanol $(12 \mathrm{~mL} / \mathrm{kg} \mathrm{bw})$ \\
Vc & Vc $(80 \mathrm{mg} / \mathrm{kg} \mathrm{bw})$ & $50 \%$ Ethanol $(12 \mathrm{~mL} / \mathrm{kg} \mathrm{bw})$ \\
Sodium selenite & Sodium selenite $(2.65 \mathrm{mg} / \mathrm{kg} \mathrm{bw})$ & $50 \%$ Ethanol $(12 \mathrm{~mL} / \mathrm{kg} \mathrm{bw})$ \\
CS/COS-M & CS/COS-M $(80 \mathrm{mg} / \mathrm{kg} \mathrm{bw})$ & $50 \%$ Ethanol $(12 \mathrm{~mL} / \mathrm{kg} \mathrm{bw})$ \\
L-Se & SeNPs-CS/COS-M $(40 \mathrm{mg} / \mathrm{kg} \mathrm{bw})$ & $50 \%$ Ethanol $(12 \mathrm{~mL} / \mathrm{kg} \mathrm{bw})$ \\
M-Se & SeNPs-CS/COS-M $(80 \mathrm{mg} / \mathrm{kg} \mathrm{bw})$ & $50 \%$ Ethanol $(12 \mathrm{~mL} / \mathrm{kg} \mathrm{bw})$ \\
H-Se & SeNPs-CS/COS-M $(120 \mathrm{mg} / \mathrm{kg} \mathrm{bw})$ & $50 \%$ Ethanol $(12 \mathrm{~mL} / \mathrm{kg} \mathrm{bw})$ \\
\hline \multicolumn{3}{c}{}
\end{tabular}

\subsection{Statistical Analysis}

In all of the experiments, data are presented as mean \pm SD. The difference between two groups was analyzed by a Student's $t$-test involving the utilization of the SPSS software program (version 17.0 for Windows). A $p$ value of less than 0.05 was considered to be statistically significant.

\section{Results and Discussion}

\subsection{Characterization of SeNPS}

Polysaccharides such as CS [10,11,32], alginate [33], sialic acid [34], and gum arabic [35] have been utilized to synthesize and stabilize SeNPs, and SeNPs with different morphologies have been obtained. It seems that polysaccharides of high molecular weight (MW) can be used to prepare and stabilize SeNPs. Herein, aqueous Se (IV) was chemically reduced by ascorbic acid (Vc) to synthesize SeNPs (elemental Se particles) in the presence of CS (37 kDa) or COS $(2.5 \mathrm{kDa})$ in order to evaluate the surface decoration of SeNPs with the positively charged polysaccharides. As a result, spherical SeNPs were initially synthesized in CS, COS, or water, though their size varied (Figure S1A-C, Supplementary Materials). However, the changes in them differed. A few hours later, the bare SeNPs that were synthesized in water without any surface decoration quickly aggregated into bulks (Figure 1A), while a change in shape and aggregation of SeNPs were found in the COS solution at $24 \mathrm{~h}$ after the initial formation stage (Figure 1B). However, highly uniform monodisperse spherical SeNPs, as presented in Figure $1 \mathrm{C}$, were finally obtained in aqueous $\mathrm{CS}$, with an orange or red appearance that was dependent on the concentration of SeNPs (Figure 1C inset). The chitosan-decorated selenium nanoparticles (CS-SeNPs) were found to remain stable at $4{ }^{\circ} \mathrm{C}$ for about 1 month without visible deposition. CS was found to be superior to COS in terms of controlling the formation, shape, and stability of SeNPs. 

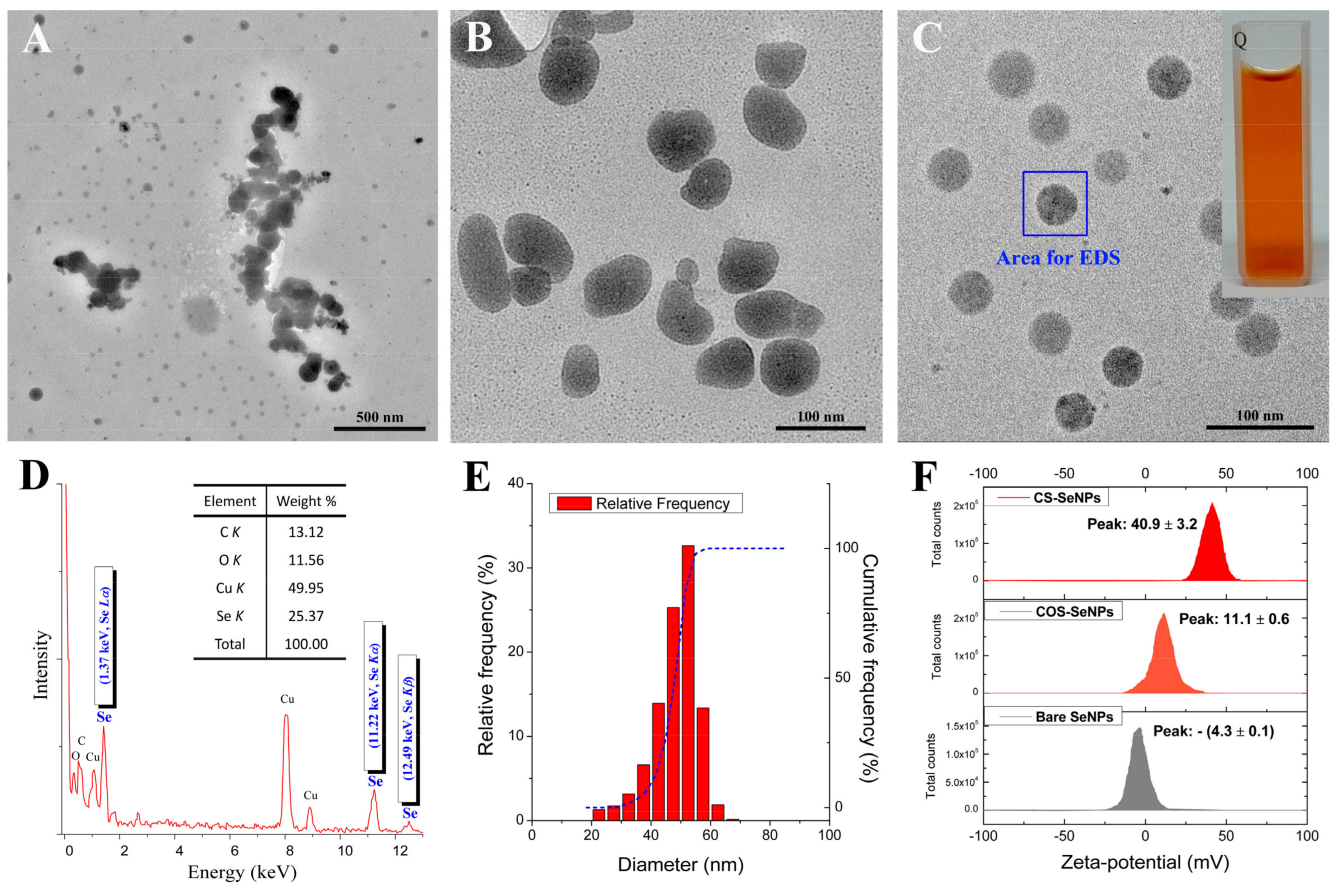

Figure 1. Typical morphology and formation of selenium nanoparticles (SeNPs). TEM images of (A) bare SeNPs, (B) chitooligosaccharide (COS)-SeNPs, and (C) chitosan (CS)-SeNPs. (D) EDS spectra of CS-SeNPs determined from the area shown in Figure 1C. (E) The size distribution of CS-SeNPs measured from the TEM results. (F) Zeta-potentials of bare SeNPs, COS-SeNPs, and CS-SeNPs, measured at a pH of $5.0 \pm 0.3$.

Some characteristics of SeNPs were also investigated. Typical Se peaks $(1.37,11.22$, and $12.49 \mathrm{keV}$, identified as Se $L_{\alpha}$, Se $K_{\alpha}$, and Se $K_{\beta}$, respectively) were found in the EDS spectra of SeNPs, confirming the elemental nature of these nanoparticles (Figure 1D). Moreover, a size distribution analysis indicated that the diameter of SeNPs could be controlled by CS within a nano-defined range (Figure 1E and Table 2). Nonetheless, the size distribution as measured by TEM (Figure 1E) and dynamic light scattering (DLS, Table 2) showed that the average diameters of the CS-SeNPs were around 50 and $100 \mathrm{~nm}$, respectively. These results suggest that an invisible layer covered the SeNPs since DLS can only measure hydrodynamic size [10,32]. Furthermore, the size and dispersity of the SeNPs were monitored over time to evaluate the stability of these nanoparticles in solution. As shown in Table 2, the fresh SeNPs had similar diameter and polydispersity index (PDI) values. The Z-average sizes of the bare SeNPs and COS-SeNPs increased over time; however, the size of the CS-SeNPs was found to be about $80 \mathrm{~nm}$ with a low PDI $(0.116 \pm 0.018)$ after 14 days of storage. The 'bottom-up' growth process of COS-SeNPs and the 'top-down' shrinkage process of CS-SeNPs over time reported by Leng et al. [32] were also found in this study, indicating that CS was able to control the size of SeNPs during common storage. The ability of CS to control the stability of SeNPs is important to the industrial production of SeNPs. 
Table 2. The dynamic light scattering (DLS) results for bare SeNPs, COS-SeNPs, and CS-SeNPs $(n=3)$.

\begin{tabular}{lcc}
\hline \multicolumn{1}{c}{ Sample } & $\begin{array}{c}\text { Z-Average Diameter } \\
(\mathbf{n m})\end{array}$ & PDI \\
\hline Bare SeNPs (fresh) & $102.9 \pm 6.4$ & $0.355 \pm 0.06$ \\
COS-SeNPs (fresh) & $97.5 \pm 3.6$ & $0.305 \pm 0.044$ \\
CS-SeNPs (fresh) & $103.3 \pm 2.3$ & $0.262 \pm 0.035$ \\
Bare SeNPs (after 6 h of storage at $25^{\circ} \mathrm{C}$ ) & $265.2 \pm 15.6$ & $0.351 \pm 0.040$ \\
COS-SeNPs (after 14 days of storage at $25^{\circ} \mathrm{C}$ ) & $335.7 \pm 11.7$ & $0.472 \pm 0.028$ \\
CS-SeNPs (after 14 days of storage at $25^{\circ} \mathrm{C}$ ) & $80.5 \pm 0.6$ & $0.116 \pm 0.018$ \\
\hline
\end{tabular}

PDI, polydispersity index.

In addition, the zeta-potential was studied at a $\mathrm{pH}$ of $5.0 \pm 0.3$. The results shown in Figure $1 \mathrm{~F}$ illustrate that the surface potential of the SeNPs was dependent on the surface decoration. To be more specific, the bare SeNPs were almost electroneutral $(-4.3 \pm 0.1 \mathrm{mV})$ in water, while the zeta-potential of SeNPs was elevated by CS to $40.9 \pm 3.2 \mathrm{mV}$, probably due to the positively charged $-\mathrm{NH}_{3}{ }^{+}$groups from the CS [10,32]. This might ensure that SeNPs remain stable during the process of dialysis or UF. COS decoration also led to an increase in the zeta-potential of the SeNPs $(11.1 \pm 0.6 \mathrm{mV})$; however, the elevation was weak and partly responsible for the weak stability of SeNPs in COS solution. Nonetheless, these results may be not be in line with a previous study [32] that reported higher stability (30 days) and zeta-potentials $(>45 \mathrm{mV}$ ) for SeNPs when these nanoparticles were modulated by CS (3 and $200 \mathrm{kDa}$ ) at a $\mathrm{pH}$ of $3.2 \pm 0.3$. This result could be partly attributed to the fact that the zeta-potential might change with $\mathrm{pH}$ [35]. A lower $\mathrm{pH}$ may result in a higher zeta-potential, as more $-\mathrm{NH}_{2}$ groups might become $-\mathrm{NH}_{3}{ }^{+}$groups, which may be beneficial to the stability of SeNPs. A previous study [32] reported that CS was more efficient than COS at elevating the zeta-potential of SeNPs. Considering the relationship between nanoparticles' stability and their zeta-potential, CS was found to be superior to COS in the preparation of stable SeNPs.

\subsection{Characterization of SeNPs-CS/COS-Ms}

Bare SeNPs and CS-SeNPs are not available for commercial application in oral administration systems due to their poor stability $[10,11]$, which is closely related to the decrease in zeta-potential during storage. SeNPs might finally become dark bulk aggregates even in the presence of CS, following an unavoidable enlargement in size $[10,36]$ and aggregation in an aqueous environment $[10,11,36]$. In our previous work, SeNPs were loaded into solid CS microspheres [10] or a chitosan/citrate complex [11] to overcome the instability of the nanoparticles, and these solid mixtures allowed for the release of SeNPs and Se supplementation in animals. Physical isolation of SeNPs in solid vehicles seems to be a feasible way to develop a Se supplement based on SeNPs $[10,11]$. However, more efficient SeNP supplementation is needed.

COS was found to be more soluble than CS because of its low MW, while CS was found to be more efficient than COS at maintaining stable SeNPs. In the present study, SeNPs, CS, and COS were mixed together in a proper proportion and the mixture was spray-dried to produce SeNPs-CS/COS-Ms. The SeNPs complex is actually a collection of microparticles with a spherical or irregularly wrinkled surface (Figure 2A). The size distribution analysis performed in ethanol indicated that the size of the microparticles was 2-20 $\mu \mathrm{m}$, while small sizes were observed in water (Figure 2B). Neither of the polysaccharides were soluble in ethanol, while COS was found to be soluble in water and CS was found to be not soluble in water $[22,37,38]$. Moreover, ultrasonic disruption of particles was conducted in absolute ethanol, and it was found that the SeNPs-CS/COS-Ms were hollow (Figure 2C). The structure was very different from that of selenium-nanoparticles-loaded chitosan microspheres (SeNPs-CS-Ms) with solid cores [10]. The wrinkled surface may partly be explained by the lack of balance between the moisture diffusion and the moisture evaporation that occurred during spray-drying [39], and partly by the weak mechanical strength of COS (and also the low MW of CS) as compared with CS [40]. As 
for the hollow core, this may have resulted from the film-formation capacity and the viscosity of the polysaccharides [37,38], which allowed for the generation of a smooth surface inside SeNPs-CS/COS-Ms. Evidently, SeNPs-CS/COS-Ms with an appreciable specific surface area were developed.
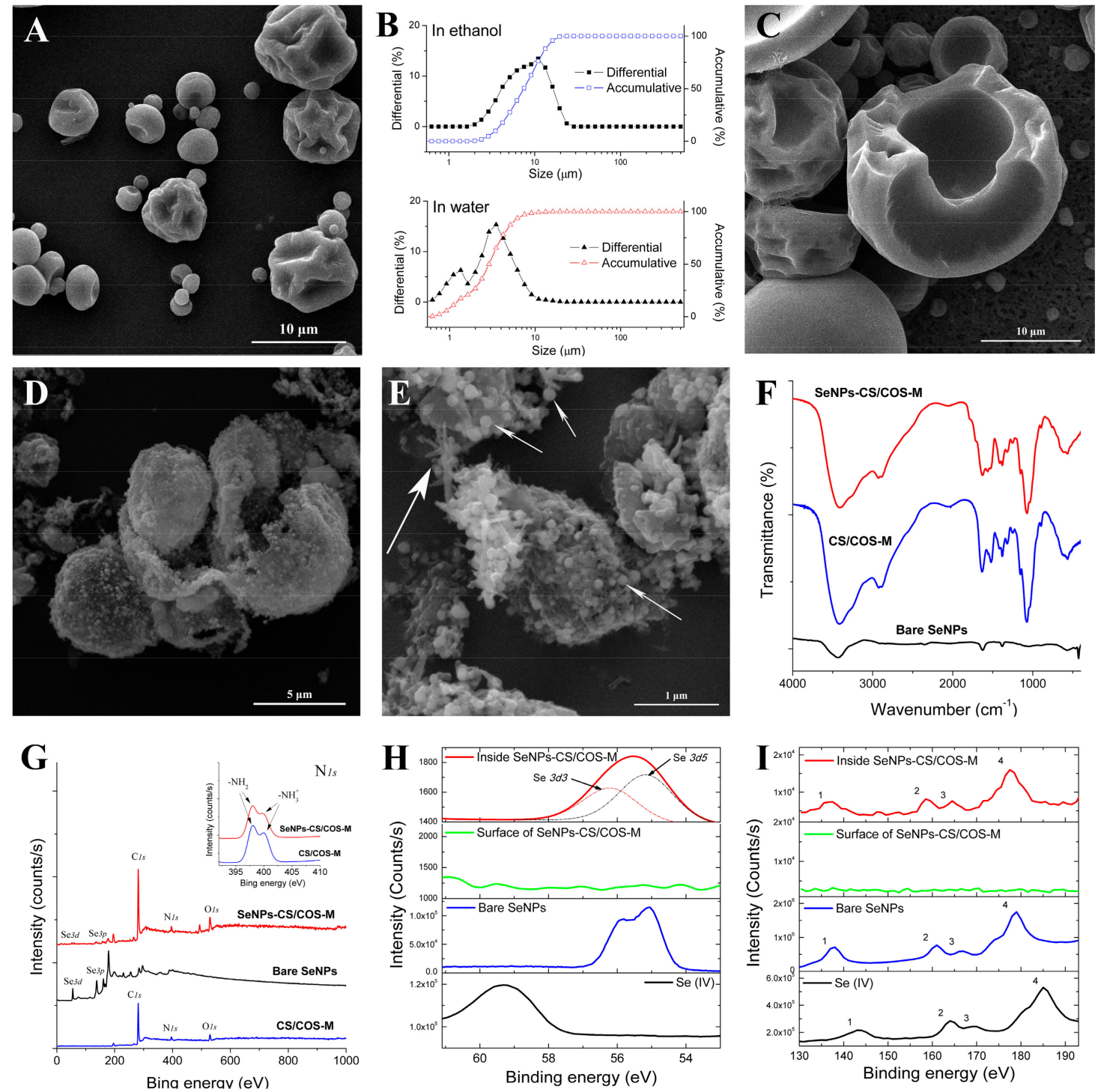

Figure 2. The typical morphology and chemical properties of selenium-nanoparticles-loaded chitosan/chitooligosaccharide microparticles (SeNPs-CS/COS-Ms).

(A) An SEM image of SeNPs-CS/COS-Ms. (B) The size distribution of SeNPs-CS/COS-Ms in ethanol (upper) and in water (lower). (C) An SEM image of SeNPs-CS/COS-Ms after ultrasonic disruption in absolute ethanol. (D-E) SEM images of SeNPs-CS/COS-Ms at different magnifications, exactly (D) 16,000× and (E) 50,000×, after ultrasonic disruption in 50\% ethanol. (F) FTIR spectra of bare SeNPs, CS/COS, and SeNPs-CS/COS-Ms. (G-I) XPS results, including (G) wide-range XPS patterns, (H) Se 3d scan XPS patterns composed of Se $3 d 3$ and Se $3 d 5$ signals, and (I) Se $3 p$ scan XPS patterns. In Panel (E), smaller arrows indicate CS-SeNP nanobeads, while the bigger one represents the linear CS structure.

Furthermore, 50\% ethanol was used to remove the COS within SeNPs-CS/COS-Ms in order to obtain more detail. The skeleton, comprised of CS and SeNPs, remained as presented in Figure 2D, since these two compounds are unable to be dissolved by $50 \%$ ethanol in theory. Nanobeads with a size of 60-150 nm were found on the remnants of the SeNPs-CS/COS-Ms, and seemed to be fixed onto some linear or lumpy structures (Figure 2E). This suggests that the space around the nanobeads was filled with COS. That is, the hollow and wrinkled SeNPs-CS/COS-Ms were actually a close layer of solid COS 
filled with the nanobeads. This allowed for the quick disintegration of SeNPs-CS/COS-Ms in water as shown in Figure 2B, which is impossible for SeNPs-CS-Ms in the same dispersant [10]. The nanobeads, whose EDS patterns were similar to that shown in Figure 1D, showed that individual SeNPs were capped by CS rather than COS during the mixing and spray-drying processes. This also implies that CS is superior to COS in terms of the surface decoration of SeNPs. The structure of SeNPs-CS/COS-Ms might facilitate the physical isolation and release of SeNPs.

Other chemical characteristics of the SeNPs-CS/COS-Ms were investigated. A FTIR analysis was conducted to explore whether any chemical modification of functional groups occurred. SeNPs-CS/COS-Ms with different material ratios were found to share comparable FTIR spectra, and they seemed to be a simple collection of SeNPs, CS, and COS, without any new functional groups found in the FTIR spectra (Figure 2F). In addition, an XPS analysis was performed to examine the status of Se. As shown in Figure 2G, the XPS signals of C, O, N, and Se were found, and the characteristic peaks of $-\mathrm{NH}_{3}{ }^{+}$and $-\mathrm{NH}_{2}$ were found in both CS/COS-M and SeNPs-CS/COS-M, which is consistent with previous studies reporting that these two chemical states of nitrogen can be observed by $\mathrm{N} 1 \mathrm{~s}$ XPS [10,11,41]. Additionally, the peaks found at $55.3 \mathrm{eV}$ and $59.5 \mathrm{eV}$ in the XPS patterns (shown in Figure $2 \mathrm{H}$ ) were identified as the typical Se $3 d$ signals of Se (0) and Se (IV), respectively, confirming that the Se within the SeNPs-CS/COS-Ms was in an elementary state $[10,11,32]$. The Se $3 d$ result observed in the SeNPs-CS/COS-Ms was actually composed of the Se $3 d 3$ and Se $3 d 5$ signals, which was also found in the bare SeNPs (Figure $2 \mathrm{H}$ ). This result implies that these chemically fabricated SeNPs are similar in terms of their molecular structure and chemical environment. However, the Se $3 d$ signals on the surface of the SeNPs-CS/COS-Ms were much weaker than those inside the microparticles, which were found by using argon ion etching to expose the elements inside. Similar results were observed when Se $3 p$ scanning was conducted, and a shift in the Se $3 p$ signals was also found when comparing Se (0) with Se (IV) (Figure 2I). Undoubtedly, most of the SeNPs were enclosed inside SeNPs-CS/COS-Ms.

\subsection{Release of SeNPs from SeNPs-CS/COS-Ms}

The release of nanoparticles from a vehicle is very important to their absorption and their bioactivities in vivo [4,11]. In the previous studies [10,11] performed by the authors, SeNPs were found to escape from their vehicles, including CS microspheres and a chitosan/citrate complex, finally resulting in Se supplementation. Nonetheless, aspects of the vehicle's composition, such as the CS to COS ratio, might affect the release of SeNPs from the vehicle in water or the digestive tract. In this study, the release of SeNPs from SeNPs-CS/COS-Ms was tested in SGF ( $\mathrm{pH} 1.5)$ and SIF ( $\mathrm{pH} 7.2$ ) by using some SeNPs-CS/COS-M samples with comparable Se content (approximately $15 \mathrm{mg} / \mathrm{g}$ ) and different COS:CS ratios $\left(25: 75,75: 25\right.$, and 90:10). After incubation in SGF or SIF at $37^{\circ} \mathrm{C}$, intact SeNPs could be found in the digestive fluids (Figure 3A), suggesting that SeNPs were released from SeNPs-CS/COS-Ms in the mammalian digestive tract. This result also implies that embedding SeNPs in CS/COS microspheres does not significantly modify SeNPs' size and morphology, which are two important properties that might profoundly affect the bioactivities of the nanoparticles [5,42].

The release of SeNPs was found to be dependent on the digestive environment and the CS:COS ratio. The release of SeNPs was found to be faster in SGF than in SIF (Figure 3B,C). This result may be due to the acid solubility of CS, which allowed for the full release of SeNPs, while only the SeNPs absorbed on the surface or those embedded by COS could escape in the SIF. This indicates that most of the SeNPs will be released in the stomach but not in the gut. In addition, the release of SeNPs was affected by the CS:COS ratio in the order 10:90 > 25:75 > 75:25 (Figure 3B). This suggests that a higher percentage of COS in SeNPs-CS/COS-Ms might benefit the release of SeNPs in the stomach, as COS is freely soluble in water in a wide $\mathrm{pH}$ range [37,38]. The wrinkled, hollow, and nanobead-loaded structure of SeNPs-CS/COS-Ms might also have contributed to the different release rates of Se Ps, because the CS-SeNP nanobeads were surrounded by COS. An increase in the COS content and the quick dissolution of COS might be beneficial to the exposure of the SeNPs to the digestive system. Moreover, a decrease in Se release was found in both SGF and SIF, though the 
decrease was very slight. This result indicates that the stability of SeNPs might be challenged by digestive fluids. When considering the residence period (commonly $1-4 \mathrm{~h}$, dependent on food) of the diet in the stomach $[43,44]$, most of the released SeNPs, however, might be stable in the gastric phase. SeNPs-CS/COS-Ms with a CS:COS ratio of $\leq 10: 90$, which enables the full release of SeNPs in a gastric environment, were optimized for further investigations.
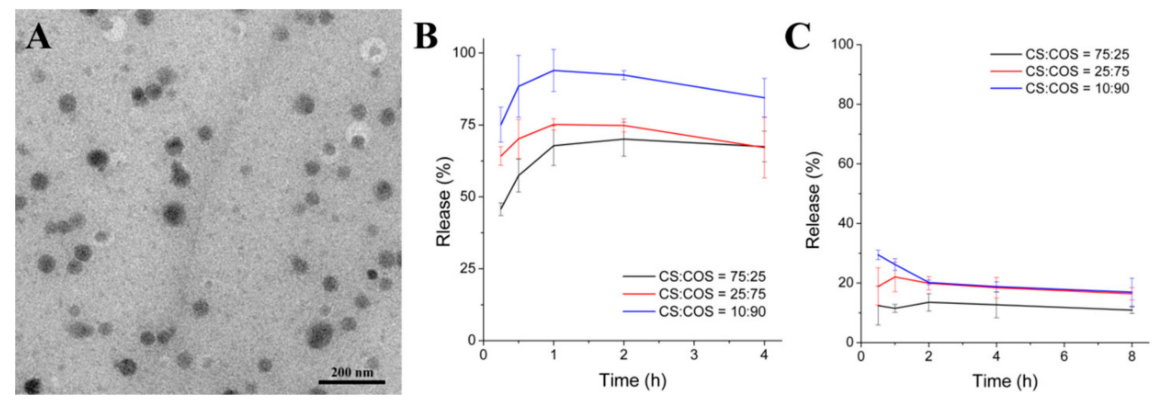

Figure 3. The release of SeNPs from SeNPs-CS/COS-Ms in simulated gastric fluid (SGF) and simulated intestinal fluid (SIF). (A) A typical TEM image of the released SeNPs in SGF or SIF. (B,C) The relative release rates of SeNPs from SeNPs-CS/COS-Ms with different material ratios of CS:COS (75:25, 25:75, and 10:90) in (B) SGF and (C) SIF.

\subsection{Free Radical Scavenging Ability of SeNPs-CS/COS-Ms}

Overconsumption of alcohol can produce an excessive amount of radical oxygen species (ROS), which can lead to oxidative damage to the lipids and proteins in tissues $[17,20]$. Here, -DPPH and $\bullet \mathrm{O}_{2}{ }^{-}$were used as model free radicals to investigate the radical scavenging ability of SeNPs-CS/COS-Ms. Considering the acceptable release of SeNPs-CS/COS-Ms when their COS:CS ratio is high, SeNPs-CS/COS-Ms with a CS:COS ratio of 10:90 were tested. As a strong antioxidant, Vc exhibited potent radical scavenging activity against these two free radicals, while selenite did not remove the radicals (Figure 4A,B). However, SeNPs-CS/COS-Ms were able to clear $\bullet$ DPPH and $\bullet \mathrm{O}_{2}{ }^{-}$ in a dose-dependent manner, although their capacity to scavenging radicals was weaker than that of Vc (Table 3). The free radical scavenging capacity of SeNPs-CS/COS-Ms, which was found to be equal to approximately $1 / 10(\bullet \mathrm{DPPH})$ or $1 / 6\left(\bullet \mathrm{O}_{2}^{-}\right)$of that of $\mathrm{Vc}$, was acceptable.
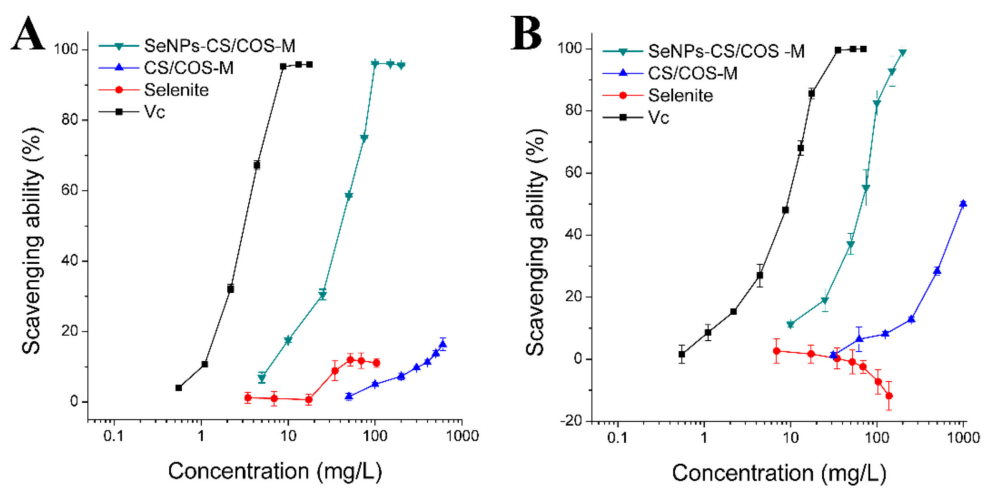

Figure 4. Free radical scavenging abilities against (A) DPPH $(\bullet \mathrm{DPPH})$ and $(\mathbf{B})$ superoxide anion $\left(\bullet \mathrm{O}_{2}{ }^{-}\right)$ radicals. SeNPs-CS/COS-Ms containing $15 \mathrm{mg}$ Se/kg (CS:COS =10:90) and CS/COS-Ms (CS:COS = 10:90) were evaluated in this section. 
Table 3. The $\mathrm{EC}_{50}$ of the free radical scavenging activities of the samples ( $\left.\mathrm{mg} / \mathrm{L}, n=3\right)$.

\begin{tabular}{ccccc}
\hline Free Radical & Vc & Sodium Selenite & CS/COS-M & SeNPs-CS/COS-M $^{\mathbf{2}}$ \\
\hline $\mathrm{DPPH}$ & $2.90 \pm 0.04$ & $>>103.8$ & $>>600$ & $29.54 \pm 1.24$ \\
\hline $\mathrm{O}_{2}{ }^{-}$ & $5.80 \pm 1.00$ & $>>138.4$ & $1082 \pm 104$ & $34.45 \pm 1.57$ \\
\hline $\begin{array}{l}\mathrm{EC}_{50} \text {, concentration for 50\% of maximal effect. } \\
\text { and the ratio of CS:COS was 10:90. }\end{array}$
\end{tabular}

The total free radical scavenging ability of SeNPs-CS/COS-Ms is the sum of the individual antioxidant capacities of their constituents, although the SeNP vehicle was considered as a whole. However, the natural activity of bare SeNPs to scavenge free radicals was difficult to determine due to its poor stability $[10,11]$. Here, the contribution of CS/COS to the free radical scavenging ability of SeNPs-CS/COS-Ms was also studied. As shown in Figure 4 and Table 3, the radical scavenging ability of CS/COS microspheres (CS/COS-Ms) was much weaker than that of SeNPs-CS/COS-Ms with the same CS:COS ratio. This result suggests that the contribution of CS or COS to the radical scavenging activity of SeNPs-CS/COS-Ms is limited. In other words, SeNPs might contribute greatly to the total radical scavenging ability of SeNPs-CS/COS-Ms.

\subsection{Acute Toxicity of SeNPs-CS/COS-Ms}

The safety of SeNPs-CS/COS-Ms should be considered before evaluating their potential in the clinic. As reported in previous work $[10,11,45]$, SeNPs are safer than selenite or selenomethionine, which are responsible for the toxicity of SeNPs/vehicle complexes. In this study, SeNPs-CS/COS-Ms were found to be much safer than selenite in terms of Se dose, with a median lethal dose $\left(\operatorname{LD}_{50}\right)$ of around 20-fold of that of sodium selenite in KM mice (Table 4). Considering the high safety of $\mathrm{CS} / \mathrm{COS}-\mathrm{Ms}\left(\mathrm{LD}_{50}>20 \mathrm{~g} / \mathrm{kg}\right.$ bw) and the low content of SeNPs $(15.1 \mathrm{mg} / \mathrm{g})$ within SeNPs-CS/COS-Ms, SeNPs might contribute greatly to the total toxicity of SeNPs-CS/COS-Ms. This result is consistent with our previous study [10] reporting that 18 -fold of the $\mathrm{LD}_{50}$ (as compared with selenite) was achieved in Institute of Cancer Research (ICR) mice by embedding SeNPs into CS microspheres. Also, SeNPs loaded on bovine serum albumin (BSA) [45] or a chitosan/citrate complex [11] yielded a similar outcome. SeNPs loaded on a protein or polysaccharide may have comparable toxicity when (1) they share similar basic physicochemical properties [46,47], such as shape, size, chemical composition, and surface properties, and (2) they can escape from their vehicles in the stomach [11]. In addition to the recorded cumulative mortality, mice were dissected to investigate the damage to the body caused by the oral administrations. Hemorrhagia points in the lungs and an abnormal liver with a rough surface were found in the dead mice given selenite or SeNPs-CS/COS-Ms (Figure S2, Supplementary Materials), indicating that these two Se forms targeted the lungs and liver. However, normal organs in the surviving mice were found at the end of the 14-day acute toxicity test. This result suggests that the damage caused by SeNPs-CS/COS-Ms can be lessened over time. Taken together, these results suggest that SeNPs, when loaded onto CS/COS-based microspheres, could be used in the clinic due to their low toxicity and good release.

Table 4. Results of the acute lethal test by a single oral administration in KM mice $(n=10)$.

\begin{tabular}{|c|c|c|c|}
\hline Sample & Se Content (mg/g) & $\mathrm{LD}_{50}{ }^{\#}(\mathrm{mg} / \mathrm{kg} \mathrm{bw})$ & $\mathrm{LD}_{50}(\mathrm{Se}) *(\mathrm{mg} \mathrm{Se} / \mathrm{kg} \mathrm{bw})$ \\
\hline Sodium selenite $^{1}$ & 456.7 & $19.2(16.2-22.7)^{\$}$ & $8.8(7.4-10.4)$ \\
\hline CS/COS-M & - & $>20 \times 10^{3}$ & - \\
\hline SeNPs-CS/COS-M & 15.1 & $11.06 \times 10^{3}\left(6.28 \times 10^{3}-19.51 \times 10^{3}\right)^{\$}$ & $167(94.9-294.5)^{\wedge}$ \\
\hline
\end{tabular}




\subsection{Protection of SeNPs-CS/COS-Ms against Oxidative Stress Induced by Alcohol}

\subsubsection{Influence of SeNPs-CS/COS-Ms on Growth}

Male KM mice were orally administered SeNPs-CS/COS-Ms at the doses of 40, 80, and $120 \mathrm{mg} / \mathrm{kg}$ bw (equal to $0.36 \%, 0.72 \%$, and $1.09 \%$ of the $\mathrm{LD}_{50}$, respectively) before the $50 \%$ ethanol challenge test. The body weight was recorded to monitor the growth of the animals. As shown in Table 5 and Figure S3, little difference in growth was found among the mice throughout the experiment. The behavior and appearance of the mice in the other groups were similar to those in the normal control group, which was given normal saline. The tested compounds, including Vc, sodium selenite, CS/COS-Ms, and SeNPs-CS/COS-Ms, at their respective doses seemed to be safe to the mice during the experiment.

Table 5. Body weight of KM mice $(n=10)$.

\begin{tabular}{cccc}
\hline \multirow{2}{*}{ Group } & \multicolumn{2}{c}{ Body Weight (g) } & \multirow{2}{*}{ Increase (\%) } \\
\cline { 2 - 3 } & Day 1 & Day 35 & \\
\hline Control & $22.93 \pm 1.45$ & $43.56 \pm 1.20$ & 89.97 \\
Model & $23.08 \pm 0.79$ & $44.84 \pm 3.63$ & 94.28 \\
Vc & $23.38 \pm 0.85$ & $43.33 \pm 3.98$ & 85.33 \\
Sodium selenite & $22.85 \pm 1.23$ & $41.36 \pm 4.18$ & 81.01 \\
CS/COS-M & $22.55 \pm 1.12$ & $42.73 \pm 3.94$ & 89.49 \\
L-Se & $22.92 \pm 0.66$ & $44.10 \pm 2.41$ & 92.41 \\
M-Se & $23.12 \pm 0.47$ & $43.21 \pm 3.85$ & 86.89 \\
H-Se & $21.93 \pm 1.05$ & $41.21 \pm 2.22$ & 87.92 \\
\hline
\end{tabular}

\subsubsection{Se Retention by SeNPs-CS/COS-Ms}

A low-Se diet $(<0.1 \mathrm{mg}$ Se $/ \mathrm{kg}$ ) may lead to Se deficiency in the body, and Se supplements can improve the Se level $[10,11,48,49]$. In this study, the serum Se retention in mice was found to be improved by selenite and SeNPs-CS/COS-Ms, while Vc and CS/COS-M might have also contributed to the Se deposition (Figure 5). It was evident that (1) SeNPs-CS/COS-Ms contributed to Se deposition more than CS/COS-M ( $p<0.05)$, (2) Se retention occurred in a dose-dependent manner, and (3) the Se deposition of all of the SeNPs-CS/COS-Ms was comparable to that of selenite $(p>0.05)$ when their Se doses were equal (1.21 mg Se/kg bw). This implies that the SeNPs within the SeNPs-CS/COS-Ms made an important contribution to the Se retention. This result is consistent with that of our previous study [10] reporting that SeNPs, rather than their CS vehicle, contributed to the capacity of SeNPs-CS-Ms to supplement Se.

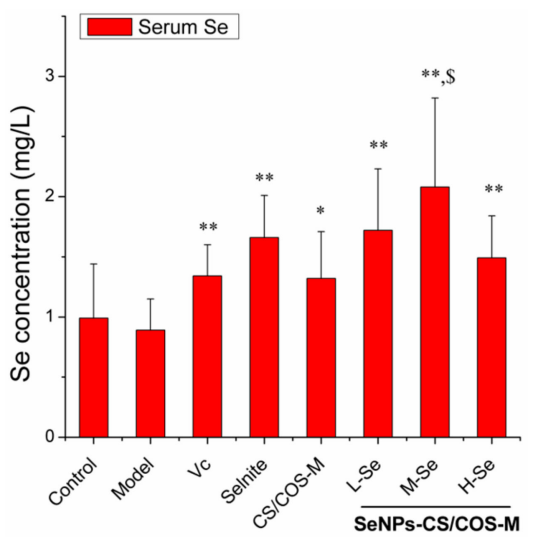

Figure 5. The serum Se retention within KM mice. The dose of each group is presented in Table 1. ${ }^{*} p<0.05,{ }^{* *} p<0.01$ versus the Model group; ${ }^{\$} p<0.05$ versus the CS/COS microsphere (CS/COS-M) group. 
The SeNPs-CS/COS-Ms and selenite had comparable Se retention capacity, while selenite produced a higher level of serum Se in mice than the SeNPs-CS-Ms did [10]. It seems that the delivery of SeNPs by using the CS/COS system might benefit Se retention. This might partly be due to the improvement in the release of free SeNPs in the digestive tract when a high percentage of COS is introduced (Figure 3). Besides this, the dose of SeNPs needs more attention. The selenite and SeNPs were found to actually be the main Se supplement, in consideration of the extremely low Se content in the feed $(<0.1 \mu \mathrm{g}$ Se/g of diet) and the estimated daily intake of adult KM mice (about 4-8 g of diet/day each) [50]. These two Se sources might be at 'super-nutritional' levels, according to Barnes [48] and Raines [49]. This might be also be confirmed by the low statistical significance of the Se levels among the L-, M-, and H-Se groups (Figure 5). SeNPs-CS-Ms at the dose of $40 \mathrm{mg} / \mathrm{kg}$ bw (equally $0.6 \mathrm{mg} \mathrm{Se} / \mathrm{kg}$ bw) were able to meet the requirement for adequate or super-nutritional Se. Thus, they deserve further investigation.

\subsubsection{Biomarkers}

Excessive consumption of alcohol can lead to severe oxidative stress in animals and humans due to its toxicity and its ability to induce $\operatorname{ROS}$ in vivo $[17,20,30,31]$. Here, the antioxidant potential of SeNPs-CS/COS-Ms was evaluated in KM mice suffering from an alcohol challenge, and some biomarkers relevant to the antioxidant activity of SeNPs-CS/COS-Ms were investigated. As shown in Tables 6 and 7, oral administration of alcohol resulted in strong oxidative stress characterized by an increase in TBARS and TCC, suggesting that oxidative damage occurred to the lipids and proteins in cells [51,52]. Alcohol was also found to decrease the levels of some protective factors, including GSH, GSH-Px, SOD, and CAT. However, SeNPs-CS/COS-Ms at the studied doses reduced TBARS and TCC, maintained GSH, and upregulated the levels of GSH-Px, SOD, and CAT, when compared with the model group given ethanol $(p<0.05$ or $p<0.01)$. These results indicate that the SeNPs-CS/COS-Ms have a powerful antioxidant ability that is comparable to that of $\mathrm{Vc}_{\mathrm{c}}$ (both $80 \mathrm{mg} / \mathrm{kg} \mathrm{bw}$ ).

Table 6. Serum biomarkers of KM mice $(n=10)$.

\begin{tabular}{ccccc}
\hline Group & TBARS $(\mathbf{n m o l} / \mathbf{m L})$ & SOD $(\mathbf{U} / \mathbf{m L})$ & GSH-PX $(\mathbf{U} / \mathbf{m L})$ & CAT $(\mathbf{U} / \mathbf{m L})$ \\
\hline Control & $10.7 \pm 4.0$ & $95.9 \pm 25.5$ & $576 \pm 160$ & $89.7 \pm 8.2$ \\
Model & $13.9 \pm 6.7$ & $82.9 \pm 26.2$ & $187 \pm 186^{\# \#}$ & $84.5 \pm 8.0$ \\
Vc & $13.1 \pm 2.4$ & $84.6 \pm 22.1$ & $431 \pm 253 *$ & $87.2 \pm 8.3$ \\
Sodium selenite & $12.8 \pm 4.6$ & $101 \pm 14$ & $343 \pm 189$ & $79.7 \pm 16.7$ \\
CS/COS-M & $11.9 \pm 4.7$ & $83.7 \pm 25.2$ & $375 \pm 285$ & $92.2 \pm 10.0$ \\
L-Se & $9.2 \pm 3.2^{*}$ & $108 \pm 10^{*}$ & $256 \pm 171$ & $84.9 \pm 9.7$ \\
M-Se & $9.2 \pm 1.8^{*}$ & $105 \pm 12^{*}$ & $451 \pm 112^{* *}$ & $88.6 \pm 9.3$ \\
H-Se & $11.8 \pm 2.5$ & $57.3 \pm 15.5^{*}$ & $437 \pm 196^{* *}$ & $72.9 \pm 21.2^{\#}$ \\
\hline \multicolumn{4}{l}{$p<0.01$ versus the Control group; ${ }^{*} p<0.05,{ }^{* *} p<0.01$ versus the Model group. }
\end{tabular}

Table 7. Hepatic biomarkers of KM mice $(n=10)$.

\begin{tabular}{cccccc}
\hline Group & $\begin{array}{c}\text { TBARS } \\
\text { (nmol/mg Prot) }\end{array}$ & $\begin{array}{c}\text { GSH } \\
\text { (mg/g Prot) }\end{array}$ & $\begin{array}{c}\text { TCC } \\
\text { (nmol/mg Prot) }\end{array}$ & $\begin{array}{c}\text { SOD } \\
\text { (U/mg Prot) }\end{array}$ & $\begin{array}{c}\text { CAT } \\
\text { (U/mg Prot) }\end{array}$ \\
\hline Control & $3.64 \pm 2.03$ & $2.91 \pm 1.20$ & $12.6 \pm 5.8$ & $90.8 \pm 16.5$ & $105 \pm 36$ \\
Model & $3.93 \pm 2.55$ & $2.01 \pm 0.48^{*}$ & $20.1 \pm 10.1^{*}$ & $85.0 \pm 16.3$ & $69.6 \pm 47.5$ \\
Vc & $2.71 \pm 1.16$ & $3.60 \pm 1.12^{* *}$ & $14.5 \pm 4.1$ & $109 \pm 16^{* *}$ & $123 \pm 57^{*}$ \\
Sodium selenite & $3.16 \pm 1.29$ & $3.77 \pm 1.36^{* *}$ & $19.0 \pm 6.6$ & $107 \pm 16^{* *}$ & $147 \pm 43^{* *}$ \\
CS/COS-M & $3.56 \pm 0.91$ & $2.10 \pm 0.34$ & $22.7 \pm 11.3$ & $96.7 \pm 17.0$ & $90.9 \pm 36.3$ \\
L-Se & $3.04 \pm 0.77$ & $2.59 \pm 0.33^{* *}$ & $13.4 \pm 7.8$ & $110 \pm 18^{* *}$ & $128 \pm 39 * *$ \\
M-Se & $2.74 \pm 0.96$ & $2.88 \pm 0.90^{*}$ & $7.3 \pm 3.3^{* *}$ & $92.1 \pm 8.0$ & $100 \pm 50$ \\
H-Se & $1.70 \pm 0.33^{*}$ & $2.53 \pm 0.47^{*}$ & $8.9 \pm 3.5^{* *}$ & $98.2 \pm 11.1 *$ & $100 \pm 17$ \\
\hline
\end{tabular}

prot, protein. ${ }^{\#} p<0.05$ versus the Control group; ${ }^{*} p<0.05,{ }^{* *} p<0.01$ versus the Model group. 
Some points need attention. The first is the contributions of CS, COS, and SeNPs to the overall antioxidant capacity of SeNPs-CS/COS-Ms. CS/COS-Ms $(80 \mathrm{mg} / \mathrm{kg} \mathrm{bw})$ did not enhance the abovementioned biomarkers, whereas SeNPs-CS/COS-Ms containing almost an equal amount of $\mathrm{CS} / \mathrm{COS}$ were able to improve the antioxidant status in mice, as evidenced by the reduction in TBARS and TCC, the increase in GSH, and the upregulation of both SOD and GSH-Px $(p<0.05$ or $p<0.01$, versus Model). It was probably the SeNPs within SeNPs-CS/COS-Ms that contributed to the antioxidant activities of the SeNP supplement in vivo. The second point is the efficiency of SeNPs in boosting GSH-Px, which is a very important antioxidant enzyme family whose members use Se as an integral part of their catalytic sites [2,4]. As presented in Table 6, SeNPs-CS/COS-Ms at the doses of 80 and $120 \mathrm{mg} / \mathrm{kg}$ bw (equal to 1.2 and $1.8 \mathrm{mg}$ Se$/ \mathrm{kg} \mathrm{bw}$, respectively) prevented the ethanol-induced reduction of GSH-Px, whereas sodium selenite at the dose of $80 \mathrm{mg} / \mathrm{kg}$ bw (equal to $1.2 \mathrm{mg} \mathrm{Se} / \mathrm{kg}$ bw) was unable to recover the level of GSH-Px. Considering the comparable Se retention among these Se sources (Figure 5), SeNPs in the form of SeNPs-CS/COS-M were more efficient in boosting the GSH-Px level than selenite. This result is not in line with some previous studies [10,53] reporting that comparable GSH-Px levels would be obtained if the Se doses were equal among different Se sources, such as selenite [10,53], BSA-SeNPs [53], and SeNPs-CS-Ms [10]. It is possible that the Se retention by SeNPs-CS/COS-Ms (shown in Figure 4) contributed to the Se storage pool in the body [54-57], finally leading to an enhancement in GSH-Px. Overall, SeNPs-CS/COS-Ms might protect animals from ethanol-induced oxidative risk, based on their capacity to upregulate the levels of GSH-Px, SOD, and CAT.

\section{Conclusions}

Both CS and COS can be used to synthesize spherical SeNPs; however, the former are superior to the latter in stabilizing SeNPs. The SeNPs-CS/COS-Ms were a collection of microparticles with a smooth or wrinkled surface and a hollow core, and SeNPs of around $50 \mathrm{~nm}$ were embedded in the SeNPs-CS/COS-Ms. Both the physical isolation of each SeNP during storage and the successful release of these nanoparticles in the stomach were carried out by SeNPs-CS/COS-Ms. The free radical scavenging ability of SeNPs-CS/COS-Ms was found to be acceptable and much stronger than that of selenite. This Se formulation was also found to be significantly more safe than selenite, with an $\mathrm{LD}_{50}$ of about 20-fold of that of selenite in mice. SeNPs-CS/COS-Ms might retard alcohol-induced oxidative stress by attenuating TBARS and TCC as well as by enhancing the levels of GSH, GSH-Px, SOD, and CAT. In summary, this mixed CS/COS microparticles design for SeNPs opens up a new path for oral delivery of Se with higher efficacy and better biosafety. SeNPs-CS/COS-Ms are a candidate source of Se worthy of further development for nutrient supplements or even nanomedicines that aim to defend against alcohol-induced oxidative injury.

Supplementary Materials: The following are available online at http://www.mdpi.com/1999-4923/12/1/43/s1, Figure S1: The SEM images of SeNPs when they were initially synthesized in (A) water, (B) aqueous COS (2.5 kDa), and $(\mathrm{C})$ aqueous CS (37 kDa); Figure S2: Organ pathology induced by SeNPs-CS/COS-Ms or selenite in mice, with the small arrows indicating hemorrhagia points in lungs and the bigger arrow indicating the abnormal liver; Figure S3: The body weight of KM mice before ethanol challenge.

Author Contributions: Conceptualization, methodology, investigation, and data curation, K.B. and B.H.; supervision, project administration, funding acquisition, and resources, K.B.; software, validation, formal analysis, and visualization, B.H. and W.H.; writing-original draft preparation and writing-review and editing, K.B. and J.H. All authors have read and agreed to the published version of the manuscript.

Funding: This research was funded by the Scientific Research Foundation of the Third Institute of Oceanography, Ministry of Natural Resources, People's Republic of China (No. 2019014), the Scientific and Technological Projects of Fujian Province, People's Republic of China (No. 2016N0018), and the Science \& Technology Major Projects of Fujian province, People's Republic of China (No. 2014NZ0001). We also thank the Marine Economic Innovation and Development Demonstration Project, Beihai, the 15th Five-Year Plan for Economic and Social Development of the People's Republic of China (No. Bhsfs007-ss-1) for its support.

Acknowledgments: We thank Yanhua Mu from Shenyang Pharmaceutical University and Yongning Li and Yabin Dai from Fujian Health College, who greatly helped with the animal experiments. We also acknowledge Li Gu 
(Third Institute of Oceanography, MNR) and Binbin Xu and Yuanfei Wu (Xiamen University) for their assistance when performing TEM, SEM, and EDS.

Conflicts of Interest: The authors declare no conflicts of interest.

\section{Abbreviations}

\begin{tabular}{|c|c|}
\hline BSA & bovine serum albumin \\
\hline bw & body weight \\
\hline CAT & catalase \\
\hline CS & chitosan \\
\hline CS/COS-Ms & chitosan/chitooligosaccharide microparticles \\
\hline COS & chitooligosaccharide \\
\hline CS-SeNPs & chitosan-decorated selenium nanopartilcels \\
\hline DLS & dynamic light scattering \\
\hline $\mathrm{DPPH}$ & 1,1-diphenyl-2-picrylhydrazyl \\
\hline $\mathrm{EC}_{50}$ & concentration for $50 \%$ of maximal effect \\
\hline EDS & energy-dispersive X-ray spectroscopy \\
\hline GSH & glutathione \\
\hline GSH-Px & glutathione peroxidase \\
\hline ICP-MS & inductively coupled plasma mass spectrometry \\
\hline ICR & Institute of Cancer Research \\
\hline $\mathrm{KM}$ & Kunming \\
\hline $\mathrm{KMnO}_{4}$ & potassium permanganate \\
\hline $\mathrm{LD}_{50}$ & median lethal dose \\
\hline MW & molecular weight \\
\hline $\mathrm{MWCO}$ & molecular weight cut-off \\
\hline PDI & polydispersibility index \\
\hline $\mathrm{RH}$ & relative humidity \\
\hline ROS & radical oxygen species \\
\hline Se & selenium \\
\hline SEM & scanning electron microscopy \\
\hline SeNPs & selenium nanoparticles \\
\hline SeNPs-CS/COS-Ms & selenium-nanoparticles-loaded chitosan/chitooligosaccharide microparticles \\
\hline SeNPs-CS-Ms & selenium nanoparticles-embedded chitosan microspheres \\
\hline SGF & simulated gastric fluid \\
\hline SIF & simulated intestinal fluid \\
\hline SOD & superoxide dismutase \\
\hline SPF & specific-pathogen-free \\
\hline TBARS & thiobarbituric acid-reactive substances \\
\hline TCC & total carbonyl compounds \\
\hline TEM & transmission electron microscopy \\
\hline UF & ultrafiltration \\
\hline Vc & ascorbic acid \\
\hline
\end{tabular}

\section{References}

1. Navarro-Alarcon, M.; Cabrera-Vique, C. Selenium in food and the human body: A review. Sci. Total Environ. 2008, 400, 115-141. [CrossRef] [PubMed]

2. Zhang, J.; Spallholz, J.E. Toxicity of selenium compounds and nano-selenium particles. In General, Applied and Systems Toxicology, 2nd ed.; Casciano, D.A., Sahu, S.C., Eds.; John Wiley \& Sons: Hoboken, NJ, USA, 2011; pp. 787-802. [CrossRef]

3. Schrauzer, G.N.; Surai, P.F. Selenium in human and animal nutrition: Resolved and unresolved issues. A partly historical treatise in commemoration of the fiftieth anniversary of the discovery of the biological essentiality of selenium, dedicated to the memory of Klaus Schwarz (1914-1978) on the occasion of the thirtieth anniversary of his death. Crit. Rev. Biotechnol. 2009, 29, 2-9. [CrossRef] [PubMed] 
4. Hosnedlova, B.; Kepinska, M.; Skalickova, S.; Fernandez, C.; Ruttkay-Nedecky, B.; Peng, Q.; Baron, M.; Melcova, M.; Opatrilova, R.; Zidkova, J.; et al. Nano-selenium and its nanomedicine applications: A critical review. Int. J. Nanomed. 2018, 13, 2107-2128. [CrossRef] [PubMed]

5. Huang, B.; Zhang, J.; Hou, J.; Chen, C. Free radical scavenging effciency of Nano-Se in vitro. Free Radic Biol. Med. 2003, 35, 805-813. [CrossRef]

6. Tran, P.A.; Webster, T.J. Selenium nanoparticles inhibit Staphylococcus aureus growth. Int. J. Nanomed. 2011, 6, 1553-1558. [CrossRef]

7. Chen, T.; Wong, Y.S.; Zheng, W.; Bai, Y.; Huang, L. Selenium nanoparticles fabricated in Undaria pinnatifda polysaccharide solutions induce mitochondria-mediated apoptosis in A375 human melanoma cells. Colloids Surf. B 2008, 67, 26-31. [CrossRef]

8. Shi, L.; Xun, W.; Yue, W.; Zhang, C.; Ren, Y.; Shi, L.; Wang, Q.; Yang, R.; Lei, F. Effect of sodium selenite, Se-yeast and nano-elemental selenium on growth performance, Se concentration and antioxidant status in growing male goats. Small Rumin. Res. 2011, 96, 49-52. [CrossRef]

9. Hu, C.H.; Li, Y.L.; Xiong, L.; Zhang, H.M.; Song, J.; Xia, M.S. Comparative effects of nano elemental selenium and sodium selenite on selenium retention in broiler chickens. Anim. Feed Sci. Technol. 2012, 177, 204-210. [CrossRef]

10. Bai, K.; Hong, B.; He, J.; Hong, Z.; Tan, R. Preparation and antioxidant properties of selenium nanoparticles-loaded chitosan microspheres. Int. J. Nanomed. 2017, 12, 4527-4539. [CrossRef]

11. Bai, K.; Hong, B.; Hong, Z.; Sun, J.; Wang, C. Selenium nanoparticles-loaded chitosan/citrate complex and its protection against oxidative stress in D-galactose-induced aging mice. J. Nanobiotechnol. 2017, 15, 92-105. [CrossRef]

12. Zhang, J.S.; Gao, X.Y.; Zhang, L.D.; Bao, Y.P. Biological effects of a nano red elemental selenium. Biofactors 2001, 15, 27-38. [CrossRef] [PubMed]

13. Jia, X.; Li, N.; Chen, J. A subchronic toxicity study of elemental Nano-Se in Sprague-Dawley rats. Life Sci. 2005, 76, 1989-2003. [CrossRef] [PubMed]

14. Yu, J.; Yao, H.; Gao, X.; Zhang, Z.; Wang, J.F.; Xu, S.W. The Role of Nitric Oxide and Oxidative Stress in Intestinal Damage Induced by Selenium Deficiency in Chickens. Biol. Trace Elem. Res. 2015, 163, 144-153. [CrossRef] [PubMed]

15. Liu, C.P.; Fu, J.; Xu, F.P.; Wang, X.S.; Li, S. The role of heat shock proteins in oxidative stress damage induced by Se deficiency in chicken livers. Biometals 2015, 28, 163-173. [CrossRef]

16. Klaunig, J.E.; Wang, Z.; Pu, X.; Zhou, S. Oxidative stress and oxidative damage in chemical carcinogenesis. Toxicol. Appl. Pharm. 2011, 254, 86-99. [CrossRef]

17. Kim, J.H.; Park, S.H.; Nam, S.W.; Choi, Y.H. Gastroprotective Effect of Selenium on Ethanol-Induced Gastric Damage in Rats. Int. J. Mol. Sci. 2012, 13, 5740-5750. [CrossRef]

18. Salim, S. Oxidative Stress and Psychological Disorders. Curr. Neuropharmacol. 2014, 12, 140-147. [CrossRef]

19. Sadau, Y.; Adelaiye, A.B.; Magaji, R.A.; Ayo, J.O.; Mabrouk, M.A.; Isa, A.I. Role of selenium and vitamin E on gastric mucosal damage induced by water-immersion restraint stress in wistar rats. IOSR J. Pharm. Biol. Sci. 2015, 10, 34-39. [CrossRef]

20. Molina, P.E.; Hoek, J.B.; Nelson, S.; Guidot, D.M.; Lang, C.H.; Wands, J.R.; Crawford, J.M. Mechanisms of Alcohol-Induced Tissue Injury. Alcohol. Clin. Exp. Res. 2003, 27, 563-575. [CrossRef]

21. Wu, C.; Xu, Z.; Huan, K. Effects of Dietary Selenium on Inflammation and Hydrogen Sulfide in the Gastrointestinal Tract in Chickens. Biol. Trace Elem. Res. 2016, 174, 428-435. [CrossRef]

22. Rinaudo, M. Chitin and chitosan: Properties and applications. Prog. Polym. Sci. 2006, 31, 603-632. [CrossRef]

23. Dufailly, V.; Noël, L.; Guérin, T. Determination of chromium, iron and selenium in foodstuffs of animal origin by collision cell technology, inductively coupled plasma mass spectrometry (ICP-MS), after closed vessel microwave digestion. Anal. Chim. Acta 2006, 565, 214-221. [CrossRef]

24. Van Lierde, V.; Chéry, C.C.; Moens, L.; Vanhaecke, F. Capillary electrophoresis hyphenated to inductively coupled plasma-sector field-mass spectrometry for the detection of chromium species after incubation of chromium in simulated sweat. Electrophoresis 2005, 26, 1703-1711. [CrossRef] [PubMed]

25. Liu, F.; Jiang, Y.F.; Du, B.J.; Chai, Z.; Jiao, T.; Zhang, C.Y.; Ren, F.Z.; Leng, X.J. Design and characterization of controlled-release edible packaging films prepared with synergistic whey-protein polysaccharide complexes. J. Agric. Food Chem. 2013, 61, 5824-5833. [CrossRef] 
26. Zhai, X.; Zhang, C.; Zhao, G.; Stoll, S.; Ren, F.; Leng, X. Antioxidant capacities of the selenium nanoparticles stabilized by chitosan. J. Nanobiotechnol. 2017, 15, 4-15. [CrossRef]

27. Xu, B.J.; Chang, S.K.C. A comparative study on phenolic profles and antioxidant activities of legumes as affected by extraction solvents. J. Food Sci. 2007, 72, S159-S166. [CrossRef]

28. Liang, X.L.; Wang, X.L.; Li, Z.; Hao, Q.H.; Wang, S.Y. Improved in Vitro Assays of Superoxide Anion and 1,1-Diphenyl-2-picrylhydrazyl (DPPH) Radical-Scavenging Activity of Isoflavones and Isoflavone Metabolites. J. Agric. Food Chem. 2010, 58, 11548-11552. [CrossRef]

29. Bliss, C.I. The calculation of the dosage-mortality curve. Ann. Appl. Biol. 1935, 22, 134-167. [CrossRef]

30. Han, Y.; Xu, Q.; Hu, J.; Han, X.; Li, W.; Zhao, L. Maltol, a Food Flavoring Agent, Attenuates Acute Alcohol-Induced Oxidative Damage in Mice. Nutrients 2015, 7, 682-696. [CrossRef]

31. Zeng, T.; Zhang, C.L.; Zhu, Z.P.; Yu, L.H.; Zhao, X.L.; Xie, K.Q. Diallyl trisulfide (DATS) effectively attenuated oxidative stress-mediated liver injury and hepatic mitochondrial dysfunction in acute ethanol-exposed mice. Toxicology 2008, 252, 86-91. [CrossRef]

32. Zhang, C.; Zhai, X.; Zhao, G.; Ren, F.; Leng, X. Synthesis, characterization, and controlled release of selenium nanoparticles stabilized by chitosan of different molecular weights. Carbohydr. Polym. 2015, 134, 158-166. [CrossRef] [PubMed]

33. Ramamurthy, C.; Sampath, K.S.; Arunkumar, P.; Kumar, M.S.; Sujatha, V.; Premkumar, K.; Thirunavukkarasu, C. Green synthesis and characterization of selenium nanoparticles and its augmented cytotoxicity with doxorubicin on cancer cells. Bioprocess. Biosyst. Eng. 2013, 36, 1131-1139. [CrossRef] [PubMed]

34. Zheng, J.S.; Zheng, S.Y.; Zhang, Y.B.; Yu, B.; Zheng, W.; Yang, F.; Chen, T. Sialic acid surface decoration enhances cellular uptake and apoptosis-inducing activity of selenium nanoparticles. Colloid Surf. B 2011, 83, 183-187. [CrossRef] [PubMed]

35. Kong, H.; Yang, J.; Zhang, Y.; Fang, Y.; Nishinari, K.; Phillips, G.O. Synthesis and antioxidant properties of gum arabic-stabilized selenium nanoparticles. Int. J. Biol. Macromol. 2014, 65, 155-162. [CrossRef]

36. Yu, B.; Zhang, Y.; Zheng, W.; Fan, C.; Chen, T. Positive surface charge enhances selective cellular uptake and anticancer efficacy of selenium nanoparticles. Inorg. Chem. 2012, 51, 8956-8963. [CrossRef]

37. Hamed, I.; Özogul, F.; Regenstein, J.M. Industrial applications of crustacean by-products (chitin, chitosan, and chitooligosaccharides): A review. Trends Food Sci. Tech. 2016, 48, 40-50. [CrossRef]

38. Xia, W.; Liu, P.; Zhang, J.; Chen, J. Biological activities of chitosan and chitooligosaccharides. Food Hydrocolloid 2011, 25, 170-179. [CrossRef]

39. Liu, W.; Chen, X.D.; Cheng, Z.; Selomulya, C. On enhancing the solubility of curcumin by microencapsulation in whey protein isolate via spray drying. J. Food Eng. 2016, 169, 189-195. [CrossRef]

40. Hsu, S.; Whu, S.W.; Tsai, C.; Wu, Y.; Chen, H.; Hsieh, K. Chitosan as scaffold materials: Effects of molecular weight and degree of deacetylation. J. Polym. Res. 2004, 11, 141-147. [CrossRef]

41. Feng, Y.; Su, J.; Zhao, Z.; Zheng, W.; Wu, H.; Zhang, Y.; Chen, T. Differential effects of amino acid surface decoration on the anticancer efficacy of selenium nanoparticles. Dalton Trans. 2014, 43, 1854-1861. [CrossRef]

42. Peng, D.; Zhang, J.; Liu, Q.; Taylor, E.W. Size effect of elemental selenium nanoparticles (Nano-Se) at supranutritional levels on selenium accumulation and glutathione S-transferase activity. J. Inorg. BioChem. 2007, 101, 1457-1463. [CrossRef] [PubMed]

43. Clegg, M.; Shafat, A. Energy and macronutrient composition of breakfast affect gastric emptying of lunch and subsequent food intake, satiety and satiation. Appetite 2010, 54, 517-523. [CrossRef] [PubMed]

44. Willis, H.J.; Thomas, W.; Willis, D.J.; Slavin, J.L. Feasibility of measuring gastric emptying time, with a wireless motility device, after subjects consume fiber-matched liquid and solid breakfasts. Appetite 2011, 57, 38-44. [CrossRef] [PubMed]

45. Wang, H.; Zhang, J.; Yu, H. Elemental selenium at nano size possesses lower toxicity without compromising the fundamental effect on selenoenzymes: Comparison with selenomethionine in mice. Free Radic. Biol. Med. 2007, 42, 1524-1533. [CrossRef] [PubMed]

46. Rivera-Gil, P.; Jimenez de Aberasturi, D.; Wulf, V.; Pelaz, B.; Del Pino, P.; Zhao, Y.; De La Fuente, J.M.; Ruiz De Larramendi, I.; Rojo, T.; Liang, X.J.; et al. The challenge to relate the physicochemical properties of colloidal nanoparticles to their cytotoxicity. Acc. Chem. Res. 2013, 46, 743-749. [CrossRef]

47. Gil, P.R.; Oberdörster, G.; Elder, A.; Puntes, V.; Parak, W.J. Correlating physicochemical with toxicological properties of nanoparticles: The present and the future. ACS Nano 2010, 4, 5527-5531. [CrossRef] 
48. Barnes, K.M.; Evenson, J.K.; Raines, A.M.; Sunde, R.A. Transcript Analysis of the Selenoproteome Indicates That Dietary Selenium Requirements of Rats Based on Selenium-Regulated Selenoprotein mRNA Levels Are Uniformly Less Than Those Based on Glutathione Peroxidase Activity. J. Nutr. 2009, 139, 199-206. [CrossRef]

49. Raines, A.M.; Sunde, R.A. Selenium toxicity but not deficient or supernutritional selenium status vastly alters the transcriptome in rodents. BMC Genom. 2011, 12, 26-40. [CrossRef]

50. Dai, H.C.; Long, L.Q.; Zhang, X.W.; Zhang, W.M.; Wu, X.X. Cloning and expression of the duck leptin gene and the efect of leptin on food intake and fatty deposition in mice. J. Anim. Sci. 2007, 20, 850-855. [CrossRef]

51. Baltacıoğlu, E.; Yuva, P.; Aydın, G.; Alver, A.; Kahraman, C.; Karabulut, E.; Akalın, F.A. Lipid Peroxidation Levels and Total Oxidant/Antioxidant Status in Serum and Saliva from Patients With Chronic and Aggressive Periodontitis. Oxidative Stress Index: A New Biomarker for Periodontal Disease? J. Periodontol. 2014, 85, 1432-1441. [CrossRef]

52. Dalle-Donne, I.; Giustarini, D.; Colombo, R.; Rossi, R.; Milzani, A. Protein carbonylation in human diseases. Trends Mol. Med. 2003, 9, 169-176. [CrossRef]

53. Zhang, J.; Wang, H.; Yan, X.; Zhang, L. Comparison of short-term toxicity between Nano-Se and selenite in mice. Life Sci. 2005, 76, 1099-1109. [CrossRef] [PubMed]

54. Behne, D.; Alber, D.; Kyriakopoulos, A. Long-term selenium supplementation of humans: Selenium status and relationships between selenium concentrations in skeletal muscle and indicator materials. J. Trace Elem. Med. Biol. 2010, 24, 99-105. [CrossRef] [PubMed]

55. Burk, R.F.; Hill, K.E.; Motley, A.K. Plasma selenium in specific and non-specific forms. Biofactors 2001, 14, 107-114. [CrossRef] [PubMed]

56. Burk, R.F.; Hill, K.E.; Motley, A.D.; Winfrey, V.P.; Kurokawa, S.; Mitchell, S.L.; Zhang, W. Selenoprotein P and apolipoprotein E receptor-2 interact at the blood-brain barrier and also within the brain to maintain an essential selenium pool that protects against neurodegeneration. FASEB J. 2014, 28, 3579-3588. [CrossRef]

57. Burk, R.F.; Hill, K.E. Regulation of Selenium Metabolism and Transport. Annu. Rev. Nutr. 2015, 35, $109-134$. [CrossRef]

(C) 2020 by the authors. Licensee MDPI, Basel, Switzerland. This article is an open access article distributed under the terms and conditions of the Creative Commons Attribution (CC BY) license (http://creativecommons.org/licenses/by/4.0/). 